Mémoire.

\title{
LA DIROFILARIOSE HUMAINE EN ITALIE
}

\author{
S. PAMPIGLIONE*, G. CANESTRI TROTTI**, F. RIVASI $* * *$
}

RÉSUMÉ

Dans le Laboratoire de Parasitologie de la Faculté de Médecine Vétérinaire de l'Université de Bologna les auteurs ont observé, depuis 1971 , 30 cas de dirofilariose humaine (sur 101 signalés en Italie) identifiés dans la presque totalité comme étant dus à Dirofilaria repens (Railliet et Henry, 1911) nématode parasite du chien relativement fréquent en Italie. De ces 30 cas, 23 étaient à localisation sous-cutanée, 4 sous-muqueux et 3 pulmonaires. La parasitose a été presque toujours méconnue cliniquement, étant inter- prétée comme une néoplasie bénigne ou maligne ou comme un corps étranger. Le parasite a pu être reconnu grâce à l'examen des coupes histologiques. Dans 3 cas on a pu énucléer du nodule l'helminthe entier. Les auteurs ont l'impression que la parasitose est beaucoup plus fréquente chez l'homme en Italie que ce qu'il apparaît dans la littérature scientifique : certains cas, pouvant guérir spontanément et d'autres n'étant pas diagnostiqués.

\section{Summary: Human dirofilariasis in Italy.}

Since 1971, at the Laboratory of Parasitology, Faculty of Veterinary Medicine, University of Bologna, 30 cases of human dirofilariasis were observed out of the 101 cases reported in Italy. The causative agent was identified in nearly all the cases as Dirofilaria repens (Nematoda, Filarioidea) a parasite relatively common in the dog in Italy. 23 cases were localized in the subcutaneous tissue, 4 in the submucosa and 3 in the lungs. In the majority of the cases the diagnosis had been misinterpreted, the lesions being first seen as malignant or benign tumours or foreign-body granuloma. The parasite was histologically recognized. In 3 cases the parasite could be extracted intact from the nodule. The authors believe that human dirofilariasis is more frequent than reported by the relevant scientific literature in that many cases are likely to recover spontaneously while others would remain undetected.

\section{INTRODUCTION}

Dirofilaria repens (Railliet et Henry, 1911), (Filarioidea, Onchocercidae) nématode parasite habituel du chien dans le Vieux Monde, transmis par la piqûre de Culicidés, a été identifié (Pampiglione et al., 1982a) comme agent de la dirofilariose humaine en Italie. La présence de ce Nématode avait déjà été signalée chez l'homme en Russie par Skrjabin et al. (1930) et son identité avec D. conjunctivae (Addario, 1885) avait été suspectée par Desportes (1939-1940), par Harant et Brès (1946), discutée par Faust (1957) et acceptée par Dissanaike (1979).

Depuis 1971 dans notre Laboratoire on a observé 30 cas de cette parasitose (sur 101 signalés en Italie) dont 23 à localisation sous-cutanée, 4 sous-muqueux et 3 pulmonaires. Parmi ces derniers, dans 2 cas le diagnostic spécifique n'a

* Cattedra di Parassitologia, Facoltà di Medicina Veterinaria, Università di Bologna, via San Giacomo 9/2, 40126 Bologna, Italie.

** Cattedra di Parassitologia, Dipartimento di Biologia Animale, Università di Torino.

*** Istituto di Anatomia ed Istologia Patologica, Università di Modena.

Accepté le : 10 septembre 1991. pu être posé avec certitude à cause des phénomènes régressifs des structures du nématode. Le parasite a pu être reconnu grâce à l'examen des coupes histologiques, en se basant sur ses dimensions, ses caractères morphologiques internes et sur l'aspect typiquement dentelé de sa cuticule. Dans 3 cas on a pu énucléer de la lésion l'helminthe entier et analyser ainsi ses caractères morphologiques, après éclaircissement au lactophénol d'Ammann, en les confrontant avec des exemplaires prélevés chez le chien.

De l'analyse des cas par nous observés (qui représentent le plus nombreux ensemble des cas étudiés par un seul Laboratoire en Europe) et des cas signalés par d'autres auteurs en Italie (tableaux I et II), on a pu tirer quelques données qu'on pense utile de reporter pour une meilleure connaissance de la parasitose, peu connue de la majorité des médecins, si non complètement ignorée.

\section{DONNÉES ANAMNÉSIQUES ET CLINIQUES}

Dans la majorité des cas l'anamnèse n'aide pas à poser le diagnostic : le patient se souvient rarement d'une piqûre d'insecte ailé qui correspond à la pénétration du parasite. La parasitose se manifeste ainsi dans la presque totalité des cas sournoisement sous l'aspect d'un nodule sous-cutané d'un diamètre de 1 à $3 \mathrm{~cm}$ (dimensions d'un noyau de cerise 
TABLEAU I. - 30 cas de dirofilariose humaine observés par les auteurs.

\begin{tabular}{|c|c|c|c|c|c|c|c|c|c|c|c|}
\hline \multirow[b]{2}{*}{ No. } & \multirow[b]{2}{*}{$\begin{array}{c}\text { Année } \\
\text { de } \\
\text { publ. }\end{array}$} & \multirow[b]{2}{*}{$\begin{array}{l}\text { Année } \\
\text { d'obs. }\end{array}$} & \multirow{2}{*}{\multicolumn{2}{|c|}{$\begin{array}{c}\text { Sexe et âge } \\
\text { du sujet }\end{array}$}} & \multicolumn{2}{|c|}{ Détails anamnestiques } & \multicolumn{5}{|c|}{ Détails morphologiques } \\
\hline & & & & & Localité $* * * *$ & $\begin{array}{l}\text { Localisation } \\
\text { du parasite }\end{array}$ & $\begin{array}{l}\text { Diamètre } \\
\text { max. }\end{array}$ & $\begin{array}{c}\text { Épaisseur } \\
\quad \text { de } \\
\text { la cuticule }\end{array}$ & $\begin{array}{c}\text { Pas de } \\
\text { la dentelure }\end{array}$ & $\begin{array}{c}\text { Hauteur } \\
\text { de } \\
\text { la dentelure }\end{array}$ & Sexe \\
\hline 1 & 1982 & 1980 & $\mathrm{~F}$ & 41 a. & Venezia Lido & avant-bras & 558 & 5 & $4,9-8,3$ & $2-2,6$ & F \\
\hline 2 & 1982 & 1981 & & $14 \mathrm{a}$. & Venezia Lido & scrotum & 396 & 6 & $6,5-8$ & $2-2,8$ & $\mathrm{~F}$ \\
\hline 3 & 1982 & 1970 & $\mathbf{M}$ & $25 \mathrm{a}$. & Pavia & avant-bras & 230 & $4,4-5$ & $6-7$ & $2,5-3$ & $\mathrm{M}$ \\
\hline 4 & 1982 & 1975 & $\mathrm{~F}$ & 66 a. * & Piacenza & mamelle & 236 & $5-5,5$ & 6,6 & 2,5 & $?$ \\
\hline 5 & 1982 & 1976 & $\mathrm{~F}$ & 67 a. $*^{*}$ & Piacenza & rég. sus-scapulaire & 262 & $3,3-4,4$ & 7 & 2,5 & $\mathrm{M}$ \\
\hline 6 & 1982 & 1978 & $\mathrm{~F}$ & 47 a. & Formigine (MO) & cou & 390 & $6,6-7,2$ & $6,5-7$ & 3 & $\mathrm{~F}$ \\
\hline 7 & 1982 & 1979 & $\mathrm{~F}$ & $38 \mathrm{a}$. & Sinalunga (SI) & rég. sous-clavière & 396 & $5-5,5$ & $6-7$ & 3 & $\mathbf{F}$ \\
\hline 8 & 1984 & 1983 & $\mathrm{~F}$ & $57 \mathrm{a}$. & Parma & poumon & $216-273$ & $9,3-11,4$ & $4-8$ & $1,5-3$ & $\mathrm{M}$ \\
\hline 9 & 1988 & 1980 & $\mathrm{~F}$ & 37 a. & Bologna & visage & 365 & $6-7$ & $8-10$ & $2-4$ & $\mathbf{M}$ \\
\hline 10 & 1988 & 1980 & $\mathrm{~F}$ & $?$ & Forli & $?$ & 395 & $6-7$ & $15-20$ & $4-6$ & $\mathbf{M}$ \\
\hline 11 & 1988 & 1983 & $\mathbf{M}$ & $60 \mathrm{a}$. & Duino (G0) & aine & 485 & $7-8$ & 10 & 2 & $\mathrm{~F}$ \\
\hline 12 & 1988 & 1983 & & 24 a. & Mestre (VE) & abdomen & 450 & 6 & $10-12$ & 2 & $\mathrm{~F}$ \\
\hline 13 & 1988 & 1983 & & $62 \mathrm{a}$. & Carpi (MO) & base thoracique & $350-500$ & $6-8$ & $8-11$ & $2-3$ & $\mathrm{M}-\mathrm{F}$ \\
\hline 14 & 1988 & 1984 & & 78 a. & Modena & mamelle & 450 & $5-7$ & $8-15$ & $2-3$ & $\mathrm{~F}$ \\
\hline 15 & 1988 & 1984 & M & $46 \mathrm{a}$. & Cervia (RA) & cuir chevelu & 570 & $5-6$ & $7-10$ & $2-3$ & $\mathrm{~F}$ \\
\hline 16 & 1988 & 1984 & $\mathrm{~F}$ & 51 a. & Reggio Emilia & abdomen & 480 & $5-8$ & $7-11$ & $2-3$ & $\mathrm{~F}$ \\
\hline 17 & 1988 & 1985 & $\mathrm{~F}$ & 35 a. & Mirandola (MO) & cuir chevelu & 260 & $4-6$ & $9-10$ & $2-3$ & $\mathbf{M}$ \\
\hline 18 & 1988 & 1986 & & 21 a. & Genova & aine & 430 & $5-7$ & $10-12$ & $2-4$ & $\mathrm{~F}$ \\
\hline 19 & 1988 & 1986 & & $41 \mathrm{a}$. & Napoli & bras & 500 & $6-8$ & $10-16$ & $3-5$ & $\mathrm{~F}$ \\
\hline 20 & 1988 & 1986 & & 52 a. & Mestre (VE) & rég. sous-mammaire & 307 & $5-7$ & $7-12$ & $1-3$ & $\mathrm{M}$ \\
\hline 21 & 1988 & 1987 & $\mathbf{M}$ & 51 a. & Mogliano V. (TV) & pharynx & 400 & $6-8$ & $9-11$ & $2-5$ & $\mathrm{~F}$ \\
\hline 22 & 1988 & 1987 & $\mathbf{M}$ & 51 a. & Ravenna & conjonctive bulb. & 450 & - & $10-12$ & - & $\mathrm{F}$ \\
\hline 23 & 1989 & 1985 & $\mathrm{~F}$ & 38 a. & Quarto S.E. (CA) & paupière inf.re & 456 & $6-7$ & $10-20$ & $2-4$ & $\mathrm{~F}$ \\
\hline 24 & 1990 & 1990 & $\mathrm{M}$ & $65 \mathrm{a}$. & Pineto (TE) & paupière sup.re & 270 & $6-8$ & $10-15$ & $2-4$ & $\mathrm{~F}$ \\
\hline 25 & 1991 & 1990 & $\mathbf{M}$ & 23 a. & Pesaro & abdomen & 475 & $14-35$ & $7-12$ & - & $\mathrm{F}$ \\
\hline 26 & 1991 & 1990 & M & $66 \mathrm{a}$. & $\begin{array}{l}\text { S. Dona di } \\
\text { Piave (VE) }\end{array}$ & poumon & 285 & $8-15$ & $8-15$ & 2 & $\mathbf{M}$ \\
\hline 27 & $1991 * *$ & 1988 & $\mathrm{~F}$ & 52 a. & Modena & rég. sacro-coccigienne & 446 & $7-12$ & $8-14$ & $1,8-2,2$ & $\mathrm{~F}$ \\
\hline 28 & $1991 * *$ & 1989 & $\mathrm{M}$ & 56 a. & Venezia Lido & rég. frontale & 358 & $18-24$ & $9-12$ & 2 & $\mathrm{~F}$ \\
\hline 291 & $1991 * * * *$ & 1990 & $\mathbf{M}$ & $58 \mathrm{a}$. & Mesola (FE) & poumon & 148 & $5-18$ & $6-10$ & $1-1,5$ & $\mathrm{~F}$ \\
\hline 30 & $1991 * *$ & 1990 & $\mathrm{~F}$ & $67 \mathrm{a}$. & Valmadonna (AL) & bras & 370 & $20-30$ & $8-15$ & $3-4$ & $\mathrm{~F}$ \\
\hline
\end{tabular}

* Même sûjet, une année après.

** Pas encore publié.

*** En majuscules les indicatifs des provinces $: \mathrm{AL}=$ Alessandria $; \mathrm{CA}=$ Cagliari $; \mathrm{FE}=$ Ferrara $; \mathrm{GO}=$ Gorizia $; \mathrm{MO}=\mathrm{Modena}$ $\mathrm{RA}=$ Ravenna; $\mathrm{SI}=$ Siena $; \mathrm{TE}=$ Teramo; $\mathrm{TV}=$ Treviso $; \mathrm{VE}=$ Venezia .

**** Cas déjà signalé par Fabbretti et al. (1990).

à une grosse noix), rarement plus volumineux (Storace, 1963) de consistance dure-élastique, peu mobile et peu douloureux à la palpation. Dans 3 cas seulement le contenu était purulent (Tecce, 1908, Cancrini et al., 1988 et le cas $\mathrm{n}^{\circ} 30$ de notre série au tableau I). S’il est localisé sous la conjonctive le parasite peut être visible, plus ou moins enroulé en spirale et mobile (Cancrini et Accardi, 1980; Pampiglione et al., 1989; Garavelli et al., 1990), ou encapsulé dans une espèce de kyste connectival (Addario, 1885, Cirincione, 1900). Dans un cas le parasite était logé dans le tissu musculaire du grand pectoral (Bartoli, 1950). Dans les cas pulmonaires il était toujours dans la lumière d'une artériole, au centre d'une zone d'infarctus pulmonaire (Pampiglione et al., 1984a; Pampiglione et al., 1984b; Pampiglione et al., 1991b; Fabbretti et al., 1990; Pampiglione e Fedeli, 1991).

Les localisations plus fréquentes (fig. I) sont dans la moitié supérieure du corps (80\% sur 101 cas); 32 localisés à la tête (dont 18 dans la région de l'œil, paupières et conjonctives), 24 à la paroi thoracique (dont 9 dans la mamelle), 19 au membre supérieur, 1 au cou, 1 au pharynx et 3 pulmonaires. Pour la moitié inférieure du corps on a constaté 7 localisations au membre inférieur, 8 dans la paroi abdominale, 3 dans le scrotum, 1 à la verge, 1 dans la région sacro-coccigienne. Dans un de nos cas on n'a pas reçu d'indication sur la localisation du nodule envoyé par le chirurgien.

Dans le lieu primitif de pénétration, le nématode peut rester bloqué par la réaction inflammatoire comme il paraît démontré par les rares cas dans lesquels le patient se souvient d'une piqûre d'insecte à l'endroit du nodule (Colla et Fontana, 1967; Scafa et al., 1985) et dans les cas de notre série $n^{\circ} 6$ et 14 (Pampiglione et al., $1982 b$; Pampiglione et al., 1988); ou il peut migrer vers un autre siège, 
TABLEAU II. - Cas de dirofilariose humaine signalés en Italie par d'autres auteurs.

\begin{tabular}{|c|c|c|c|c|c|}
\hline Année & Auteur & $\begin{array}{r}\text { Sexe } \\
\text { du }\end{array}$ & $\begin{array}{l}\text { et âge } \\
\text { sujet }\end{array}$ & Localité* & Localisation \\
\hline 1864 & Pane & M & $20 \mathrm{a}$. & Napoli & lèvre sup.re \\
\hline 1866 & Pace & M & 9 a. & Mirto (NA) & paupière sup.re \\
\hline 1885 & Addario & $\mathrm{F}$ & $70 \mathrm{a}$. & Brucoli (CT) & conjonctive bulbaire \\
\hline 1887 & $\begin{array}{l}\text { Grassi } \\
\text { (Condorelli-Francaviglia, 1892) }\end{array}$ & $\mathrm{M}$ & $?$ & Milano & œil (conjonctive?) \\
\hline 1900 & $\begin{array}{l}\text { Supino } \\
\text { (Cirincione, 1900) }\end{array}$ & $\mathrm{F}$ & $17 \mathrm{a}$. & Ficarazzi (CT) & conjonctive bulbaire \\
\hline 1906 & Alessandrini & $\mathrm{F}$ & $25 \mathrm{a}$. & Roma & avant-bras \\
\hline 1908 & $\begin{array}{l}\text { Pierantoni } \\
\text { (Tecce, 1908) }\end{array}$ & M & $20 \mathrm{a}$. & Napoli & doigt de la main \\
\hline 1930 & Dolfini & $\mathrm{F}$ & 27 a. & Dolo (VE) & paupière sup.re \\
\hline 1937 & Babudieri & M & $18 \mathrm{a}$. & Pieris (TS) & joue \\
\hline 1943 & Rossi & $\mathrm{F}$ & $36 \mathrm{a}$. & Carisio (VC) & mamelle \\
\hline 1950 & Bartoli & $\mathrm{F}$ & 44 a. & Mestre (VE) & rég. pectorale \\
\hline 1950 & Bevacqua, Wirth & $\mathrm{F}$ & $54 \mathrm{a}$. & Casteggio (PV) & cavité orbitaire \\
\hline \multirow[t]{3}{*}{1953} & Rossi, Genesi & $\mathrm{F}$ & $53 \mathrm{a}$. & Trino V. (VC) & mamelle \\
\hline & Rossi, Genesi & $\mathrm{F}$ & 29 a. & Villata (VC) & rég. pectorale \\
\hline & Rossi, Genesi & $\mathrm{F}$ & $28 \mathrm{a}$. & Vercelli & mamelle \\
\hline 1963 & $\begin{array}{l}\text { Ricci } \\
\text { (Storace, 1963) }\end{array}$ & $\mathrm{F}$ & $31 \mathrm{a}$. & Vidigulfo (PV) & hypocondre \\
\hline \multirow[t]{2}{*}{1967} & Colla, Fontana & $\mathbf{M}$ & $71 \mathrm{a}$. & Ghiarole (RE) & joue \\
\hline & Colla, Fontana & $\mathrm{F}$ & $54 \mathrm{a}$. & Brescello (RE) & joue \\
\hline \multirow{4}{*}{1968} & Bianchi & $\mathrm{F}$ & $40 \mathrm{a}$. & Stradella (PV) & bras \\
\hline & Bianchi & $\mathbf{M}$ & $26 \mathrm{a}$. & Travacò S. (PV) & rég. pectorale \\
\hline & Bianchi & M & adulte & Pavia & avant-bras \\
\hline & Bianchi & $\mathbf{M}$ & $20 \mathrm{a}$. & Certosa P. (PV) & rég. zygomatique \\
\hline \multirow[t]{4}{*}{1970} & Malfatti et al. & $\mathrm{F}$ & $45 \mathrm{a}$. & Colle C. (LU) & mamelle \\
\hline & Malfatti et al. & $\mathrm{F}$ & 39 a. & Porcari (LU) & rég. temporale \\
\hline & Malfatti et al. & $\mathbf{M}$ & $43 \mathrm{a}$. & Porcari (LU) & abdomen \\
\hline & Malfatti et al. & $\mathrm{F}$ & $57 \mathrm{a}$. & Porcari (LU) & cuisse \\
\hline \multirow{2}{*}{1972} & Bianchi, Caprioglio & $\mathrm{F}$ & $65 \mathrm{a}$. & Travacò S. (PV) & avant-bras \\
\hline & Bianchi, Caprioglio & $\mathrm{F}$ & $50 \mathrm{a}$. & Vercelli & mamelle \\
\hline \multirow[t]{4}{*}{1972} & Bonu, Zina & M & $47 \mathrm{a}$. & Alessandria prov. & rég. périorbitaire \\
\hline & Bonu, Zina & $\mathrm{F}$ & 49 a. & Alessandria prov. & paupière inf.re \\
\hline & Bonu, Zina & $\mathbf{M}$ & $45 \mathrm{a}$. & Alessandria prov. & verge \\
\hline & Bonu, Zina & $?$ & $?$ & Alessandria prov. & avant-bras \\
\hline \multirow[t]{2}{*}{1973} & Foresi, Caroli & $\mathrm{F}$ & 32 a. & Pontedera (PI) & mamelle \\
\hline & Foresi, Caroli & $\mathrm{F}$ & $40 \mathrm{a}$. & Pontedera (PI) & pli du coude \\
\hline \multirow[t]{2}{*}{1974} & Pazzaglia et al. & $\mathrm{F}$ & $36 \mathrm{a}$. & Vigevano (PV) & rég. pectorale \\
\hline & Pazzaglia et al. & $\mathrm{F}$ & $40 \mathrm{a}$. & Silvano P. (PV) & avant-bras \\
\hline 1975 & Pollavini, De Lalla & $\mathbf{M}$ & $25 \mathrm{a}$. & Milano & avant-bras \\
\hline 1978 & Dalla Pria et al. & $\mathrm{F}$ & 37 a. & Treviso & rég. occipitale \\
\hline 1979 & Lampertico, Bordoni & M & $45 \mathrm{a}$. & Lombardia & rég. axillaire \\
\hline 1980 & Cancrini, Accardi & $\mathrm{F}$ & $36 \mathrm{a}$. & Roma & paupière sup.re \\
\hline 1980 & Gozzi et al. & $\mathrm{M}$ & $18 \mathrm{a}$. & Palazzolo O. (BS) & coude \\
\hline \multirow[t]{9}{*}{1983} & Aloi et al. & $\mathrm{F}$ & $20 \mathrm{a}$. & S. Ambrogio (TO) & cuisse \\
\hline & Aloi et al. & $\mathrm{F}$ & $47 \mathrm{a}$. & Verolengo (TO) & cuisse \\
\hline & Aloi et al. & $\mathrm{F}$ & $54 \mathrm{a}$. & Torino & cuisse \\
\hline & Aloi et al. & $\mathrm{F}$ & 63 a. & Torino & épaule \\
\hline & Aloi et al. & $\mathrm{F}$ & $70 \mathrm{a}$. & Torino & avant-bras \\
\hline & Aloi et al. & $\mathrm{F}$ & 72 a. & Torino & genou \\
\hline & Aloi et al. & $\mathrm{F}$ & 33 a. & Torino & bras \\
\hline & Aloi et al. & $\mathrm{F}$ & 42 a. & Torino & rég. sus-pubienne \\
\hline & Aloi et al. & $\mathrm{F}$ & $54 \mathrm{a}$. & Gassino (TO) & cuisse \\
\hline 1983 & Gafa et al. & M & 57 a. & Scandiano (RE) & rég. frontale \\
\hline 1983 & Guerra et al. & $\mathrm{F}$ & $64 \mathrm{a}$. & Sassari & cavité orbitaire \\
\hline 1983 & Miracco et al. & $\mathbf{M}$ & $18 \mathrm{a}$. & Siena & scrotum \\
\hline \multirow[t]{2}{*}{1984} & Cancrini, Scaglione & $\mathrm{F}$ & $75 \mathrm{a}$. & Mazara d.V. (TP) & conjonctive bulbaire \\
\hline & Cancrini, Scaglione & $\mathrm{M}$ & $40 \mathrm{a}$. & Mazara d.V. (TP) & conjonctive bulbaire \\
\hline 1984 & Esposito et al. & M & 32 a. & Legnano (MI) & rég. sous-clavière \\
\hline \multirow[t]{2}{*}{1985} & Bolgan et al. & M & 71 a. & Venezia & scrotum \\
\hline & (Bolgan et al.) & M & $14 \mathrm{a}$. & Venezia Lido & scrotum \\
\hline \multirow{4}{*}{1985} & Fruttaldo et al. & $\mathrm{M}$ & 44 a. & Vercelli & rég. zygomatique \\
\hline & Fruttaldo et al. & M & $40 \mathrm{a}$. & Vercelli & rég. zygomatique \\
\hline & Fruttaldo et al. & M & $51 \mathrm{a}$. & Vercelli prov. & poignet \\
\hline & Fruttaldo et al. & $\mathrm{F}$ & 52 a. & Vercelli & rég. plantaire \\
\hline 1985 & $\begin{array}{l}\text { (Scaglia et al., } \\
\text { Scafa et al. }\end{array}$ & $\mathbf{M}$ & 57 a. & Castiglione P. (GR) & avant-bras \\
\hline
\end{tabular}


TABleau II (Suite).

\begin{tabular}{|c|c|c|c|c|c|}
\hline Année & Auteur & $\begin{array}{r}\text { Sexe } \\
d u\end{array}$ & $\begin{array}{l}\text { et âge } \\
\text { sujet }\end{array}$ & Localité * & Localisation \\
\hline \multirow[t]{3}{*}{1985} & Scaglia et al. & $\mathrm{F}$ & $73 \mathrm{a}$. & Vigevano (PV) & paupière sup.re \\
\hline & Scaglia et al. & M & $36 \mathrm{a}$. & Pavia & aisselle \\
\hline & Scaglia et al. & M & 38 a. & Pavia & rég. pectorale \\
\hline \multirow[t]{2}{*}{1987} & Miracco et al. & M & 37 a. & Massa M. (GR) & aisselle \\
\hline & Miracco et al. & $\mathrm{F}$ & $73 \mathrm{a}$. & Siena & rég. sus-clavière \\
\hline 1988 & Cancrini et al. & $\mathrm{F}$ & $32 \mathrm{a}$. & Sessa A. (CE) & mamelle \\
\hline 1988 & Garavelli et al. & $\mathrm{F}$ & $35 \mathrm{a}$. & Alessandria & rég. périorbitaire \\
\hline 1989 & Marconcini et al. & M & 12 a. & Livorno prov. & avant-bras \\
\hline 1990 & $\begin{array}{l}\text { Fabbretti et al. } \\
\text { (Pampiglione e Fedeli, 1991) }\end{array}$ & M & $58 \mathrm{a}$. & Mesola (FE) & poumon \\
\hline 1990 & Garavelli et al. & $\mathrm{F}$ & $65 \mathrm{a}$. & Alessandria & conjonctive bulbaire \\
\hline 1990 & (Cazzato et al.) & M & 51 a. & Mogliano V. (TV) & pharynx \\
\hline
\end{tabular}

Entre parenthèses les Auteurs qui ont décrit le même cas déjà publié par un autre auteur.

* En majuscules les indicatifs des provinces $: \mathrm{AL}=$ Alessandria $; \mathrm{BS}=$ Brescia $; \mathrm{CE}=$ Caserta $; \mathrm{CT}=\mathrm{Catania} ; \mathrm{FE}=\mathrm{Ferrara}$; $\mathrm{GR}=$ Grosseto $; \mathrm{LU}=$ Lucca $; \mathrm{MI}=$ Milan; NA = Napoli $; \mathrm{PI}=\mathrm{Pisa} ; \mathrm{PV}=$ Pavia $; \mathrm{RE}=$ Reggio Emilia; TO $=$ Torino; $\mathrm{TP}=$ Trapani $; \mathrm{TS}=$ Trieste $; \mathrm{TV}=$ Treviso $; \mathrm{VC}=$ Vercelli; $\mathrm{VE}=$ Venezia

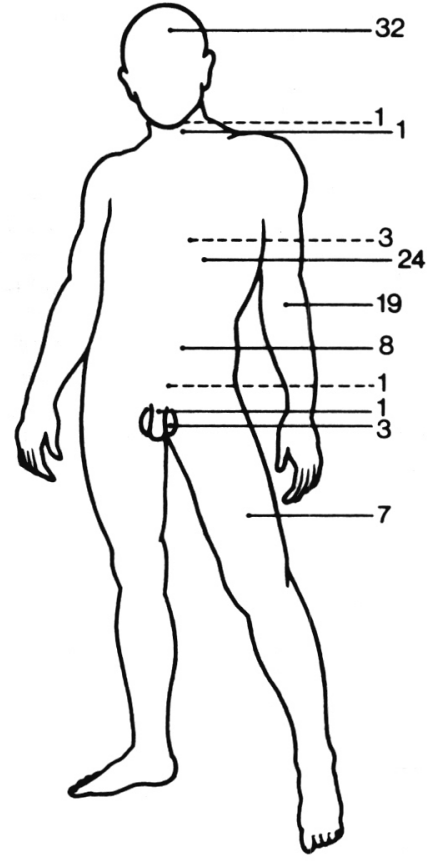

FIG. 1. - Dirofilariose humaine en Italie : localisation des cas dans l'organisme humain.

comme l'indiquent les cas 21 et 22 de notre série (Pampiglione et al., 1988; Cazzato et al., 1990) et dans l'ancien cas d'Addario (1885) où les localisations pharyngiennes ou conjonctivales ne peuvent pas représenter le point d'inoculation, vue l'extrême sensibilité de ces muqueuses, qui aurait fait déclarer aux patients la piqûre dans l'anamnèse.

D'autres démonstrations encore plus évidentes de la possibilité de déplacement du parasite du siège primitif ont été données par nos cas pulmonaires (Pampiglione et al.,
$1984 a, 1984 b, 1991 b$; Pampiglione et Fedeli, 1991), par les 2 cas de Garavelli (1988), Garavelli et al. (1990) et dans notre cas palpébral (Pampiglione et al., 1989), où le nématode a parcouru de longues migrations apparaissant en différentes parties du corps avant d'être extrait.

La symptomatologie associée peut être complètement absente ou peut se manifester par des signes inflammatoires locaux, des petites douleurs à la palpation, rarement de vraies douleurs spontanées avec ou sans irradiations, des phénomènes allergiques localisés (démangeaison, œdèmes du type de Calabar) presque jamais généralisés, urticaire). La symptomatologie peut être fugace ou se prolonger pendant des semaines ou des mois, quelquefois pendant une ou deux années (Addario, 1885; Alessandrini, 1906 ; et nos cas $n^{\circ} 5$ et 23 ).

On doit rappeler que Gardiner et al. (1978) ont décrit un cas qui s'est manifesté 8 ans après la pénétration du nématode. Dans un de nos sujets (cas 4 et 5) (Pampiglione et al., 1982b) on a eu l'apparition de 2 dirofilaires, très probablement inoculées au même moment mais avec une évolution différente vu que leur apparition dans le tissu sous-cutané a eu lieu avec une année d'écart.

Dans l'ensemble des cas les femmes apparaissent plus touchées que les hommes ( 59 contre 41 ) et les adultes plus touchés que les autres groupes d'âge (la moitié au-dessus de 44 ans) (fig. 2). Un seul cas s'est manifesté dans l'enfance ( 9 ans) et 3 dans l'adolescence (12,14 et 17 ans).

La distribution géographique dans le territoire italien semble très large (13 régions atteintes sur 20) (fig. 3) avec une prévalence plus élevée dans l'Italie du Nord (74 cas) que dans l'Italie Centrale (16 cas), le Sud (5 cas) et les îles (6 cas).

Du point de vue du diagnostic la parasitose a été presque toujours interprétée cliniquement comme une néoplasie 


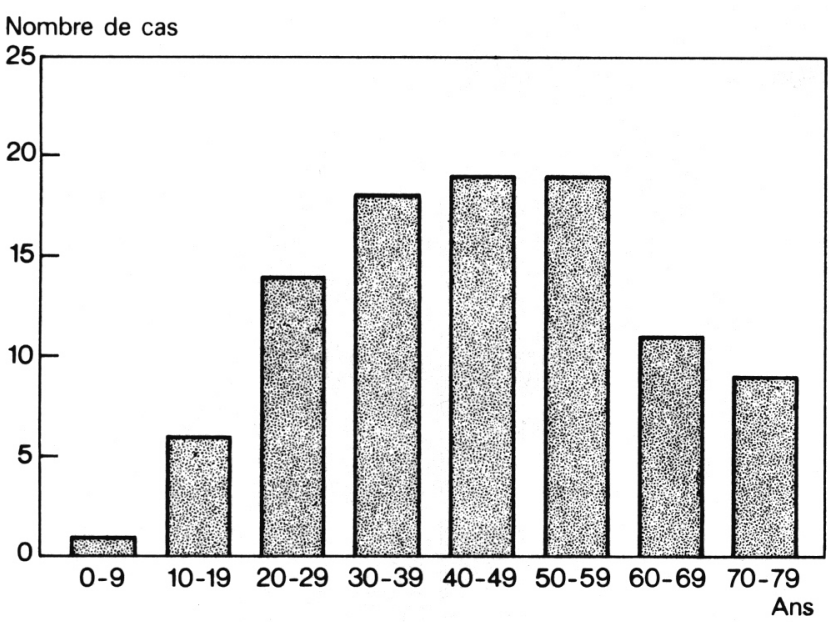

Fig. 2. - Dirofilariose humaine en Italie : distribution des cas selon l'âge (dans 4 cas l'âge n'a pas été indiqué).

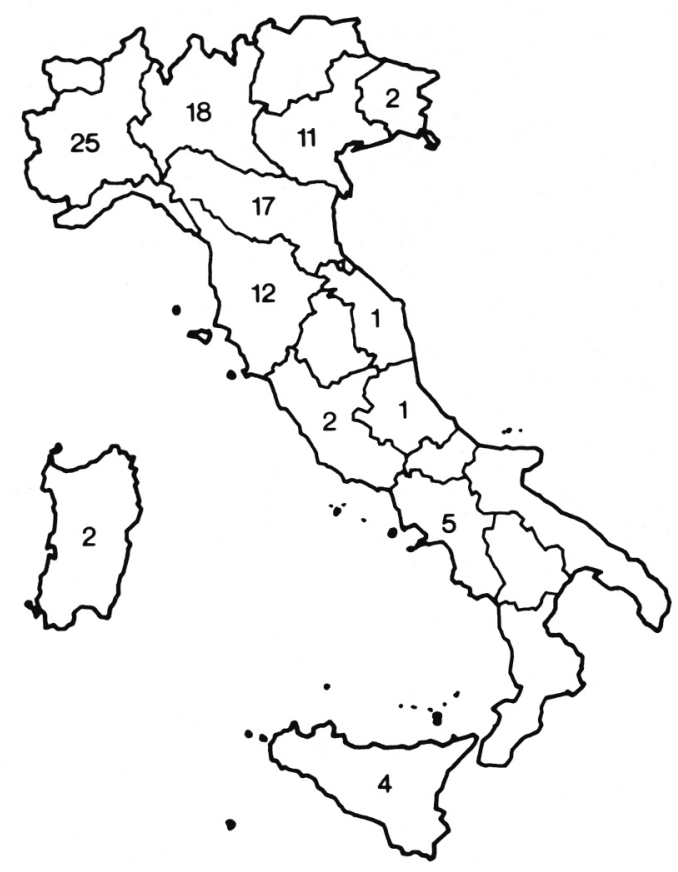

Fig. 3. - Distribution géographique

de la Dirofilariose humaine sur le territoire italien.

bénigne ou maligne ou comme un kyste sébacé, une hernie, un corps étranger. Les coupes histologiques ou l'ablation de l'helminthe entier ont permis successivement de poser le diagnostic exact. Dans le cas labial de Pane (1864) ainsi que dans le cas palpébral reporté par Dolfini (1930), c'est le patient lui-même qui a extrait le nématode piquant avec une aiguille la tuméfaction. Dans le $24^{\mathrm{e}}$ cas de notre série (Pampiglione et al., 1990) le patient en frottant rudement son nodule palpébrale a fait sortir l'helminthe spontanément.
Le pronostic est presque toujours favorable, la symptomatologie disparaît sans séquelles, après l'ablation du parasite. Font exception : le cas décrit par Addario (1885) où à la localisation sous la conjonctive bulbaire a suivi une uvéite qui a abouti à la cécité de l'œil atteint; les cas pulmonaires, où les patients ont souffert des séquelles habituelles à une segmentectomie aux dépens d'un lobe pulmonaire; le cas de mastectomie exécutée par une erreur du diagnostic (Bianchi et Caprioglio, 1972) et le cas de Bolgan et al. (1985) localisé au scrotum où également, par erreur de diagnostic, on a procédé à l'orchifuniculectomie.

Il est probable que dans plusieurs cas le nématode meurt et qu'il est détruit petit à petit par les réactions de défense immunitaires de l'hôte comme paraissent le démontrer les cas $\mathrm{n}^{\circ} 20$ et 25 de notre série (Pampiglione et al., 1984b; $1991 a$ ) où on a trouvé le parasite dans un état de dégénérescence et de fragmentation avancées.

Une réponse anticorps due à la présence de $D$. repens a été trouvée dans les quelques cas où on a fait le test de flocculation à la bentonite ou l'hémagglutination indirecte (Pampiglione et al., 1982a).

\section{Histopathologie}

Du point de vue histopathologique le processus inflammatoire apparaît toujours de type chronique, granulomateux, semblable à celui du " corps étranger », avec des micro-abcès, souvent plurinodulaires bien délimités ou à limites vagues, encerclant une ou plusieurs sections du nématode. L'infiltration inflammatoire est constituée par des granulocytes neutrophiles, éosinophiles, des cellules géantes multinucléées type Langhans et du matériel nécrotique englobant les sections du nématode. Autour de l'abcès s'épaississent des fibroblastes, des cellules épithélioïdes et des plasmocytes, formant un bourrelet de délimitation. Les éosinophiles pénètrent quelquefois dans les tissus environnants, apparemment indemnes de phénomènes inflammatoires plus marqués. Dans d'autres cas, moins nombreux, l'abcès ou les micro-abcès manquent; la réaction inflammatoire est formée d'une population cellulaire polymorphe constituée de cellules épithélioïdes, de cellules géantes multinucléées, de fibroblastes, de plasmocytes et d'éosinophiles. Rarement cette réaction inflammatoire forme de vraies structures folliculaires scléro-hyalinotiques. On peut supposer que ce dernier tableau correspond à une localisation plus ancienne du parasite, avec mort du nématode et tendance à une organisation cicatricielle (dans ces cas le parasite est fortement altéré), tandis que le premier groupe correspond à une localisation plus récente, associée à la présence du nématode souvent encore vivant. Dans les 3 cas pulmonaires, le nématode était toujours à l'intérieur d'une artériole trombosée, origine d'une petite zone d'infarctus (coin lesion, radiologiquement). 

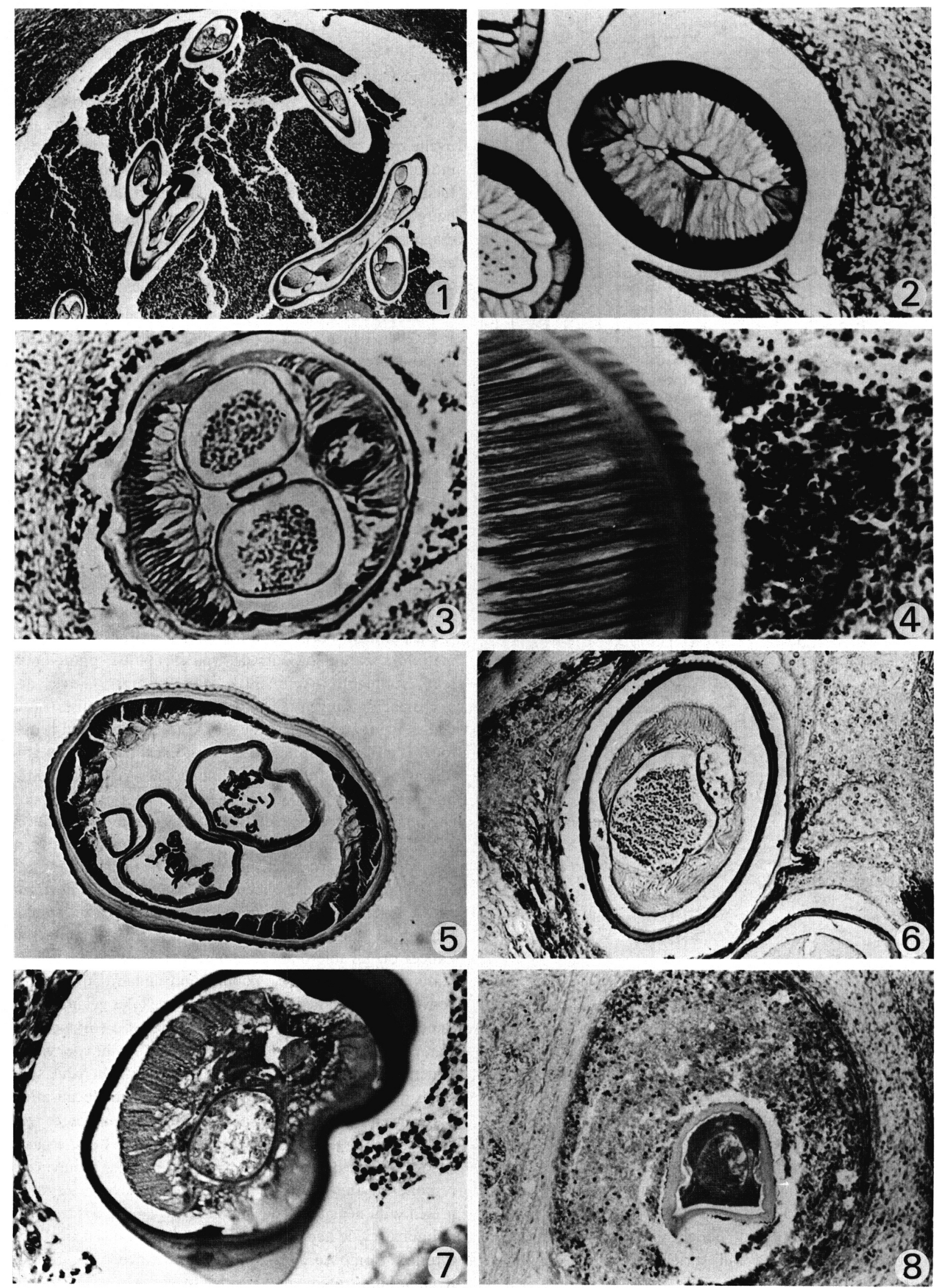


\section{PARASITOLOGIE}

Du point de vue parasitologique on note une prévalence des femelles sur les mâles (dans notre série rapport de 2:1). La présence de microfilaires dans l'utérus, notée rarement (Alessandrini, 1906; Bevacqua et Wirt, 1950; Colla et Fontana, 1967; Pampiglione et al., 1982a) fait supposer dans ces cas la présence simultanée du mâle et de la femelle. Dans les sections histologiques de notre cas $n^{\circ} 13$ (Pampiglione et al., 1988), sur la base des caractères morphologiques internes et des diamètres des sections, un mâle et une femelle semblent être présents dans le même nodule. Dans plusieurs cas les exemplaires apparaissent dans un stade d'immaturité. La présence de microfilaires dans le sang périphérique n'a jamais été notée. Les exemplaires sont dans la majorité des cas dans un bon état de conservation faisant penser qu'ils étaient vivants au moment de l'ablation du nodule. Dans plusieurs cas, en effet, l'helminthe présentait des mouvements bien évidents (Pane, 1864; Pace, 1866; Addario, 1885; Cirincione, 1900; Dolfini, 1930; Babudieri, 1937; Rossi, 1943 ; Storace, 1963; Foresi et Caroli, 1973; Dalla Pria et al., 1978; Cancrini et Accardi, 1980; Gafa' et al., 1983 ; Cancrini et Scaglione, 1984 ; Pampiglione et al., 1989, 1990; Garavelli, 1988; Garavelli et al., 1990). Les mesures du nématode dans les sections histologiques sont souvent inférieures à celles de l'helminthe examiné in toto, à cause des rétractions provoquées par l'inclusion ou par le traitement avec le xilol, etc. Dans nos cas les mesures des sections histologiques ont varié selon les données reportées dans le tableau $I$. Dans les 3 cas où on a pu examiner le vers in toto les mesures ont été les suivantes :

Cas Cas Cas

$n^{\circ} 1 \quad n^{\circ} 23 \quad n^{\circ} 25$

Longueur : $\mathrm{mm}$

$132 \quad 114 \quad 95$

Largeur $\mathrm{mx}: \mu \mathrm{m}$
Longueur œsophage : $\mu \mathrm{m}$

Anneau nerveux, distant de la bouche : $\mu \mathrm{m}$

Épaisseur de la cuticule : $\mu \mathrm{m}$

Pas de la dentelure cuticulaire : $\mu \mathrm{m}$

Vulve - distance de la bouche : $\mu \mathrm{m}$

Anus - distance de l'extrémité caudale : $\mu \mathrm{m}$
$923 \quad 915 \quad 980$

$\begin{array}{lll}285 & 228 & 275\end{array}$

$17-40 \quad 26-33 \quad 14-35$

$10-23 \quad 11-21 \quad 7-12$

$2494 \quad 2655 \quad 1900$

$110 \quad 115 \quad 135$

\section{CONCLUSIONS}

$D$. repens est largement répandu en Italie chez le chien (Pampiglione et al., 1986) avec des prévalences soit minimes, soit relativement élevées, quelquefois nettement supérieures aux infestations par D. immitis (Mantovani, 1967; Balbo et Panichi, 1968; Locatelli, 1971; Marconcini et al., 1976), espèce également répandue en Italie. Les cas humains apparaissent relativement rares : 101 cas publiés pendant une période de plus d'un siècle. Notre Laboratoire a réussi à observer 30 de ces 101 cas, après sensibilisation des chirurgiens, des dermatologistes et des histopathologistes locaux. Beaucoup d'autres cas sont vraisemblablement présents dans notre pays, mais ils passent inaperçus (non diagnostiqués ou non publiés). Le diagnostic clinique est presque toujours erroné et l'existence même de la parasitose est fréquemment ignorée des médecins. Le diagnostic exact nécessite l'examen histologique et l'identification parasitologique du nématode (techniques rarement à la disposition du médecin). Des cas où le médecin a formulé un diagnostic générique (« nodule helminthique » ne sont pas publiés (De Carneri et al., 1974). Il est également très probable que des cas d'inoculation de la larve par le vecteur ne donnent pas lieu à la formation du nodule, le nématode étant tué rapidement par la réaction tissulaire locale, et le petit gra-

(Didascalies de la planche)

Planche I. - Morphologie de Dirofilaria repens

(coupes histologiques).

1. Vue d'ensemble d'un nodule sous-cutané : il s'agit d'une femelle, sectionnée en plusieurs points (Hématoxyline/Éosine, $\times 25$ ).

2. Section transversale de femelle immature, coupée vers son extrémité caudale. On peut remarquer la diversité du profil des lignes latérales dans la section centrale et dans les deux autres limitrophes, en relation à une différente hauteur des coupes $(\mathrm{H} / \mathrm{E}, \times 250)$.

3. Section transversale de femelle adulte. On distingue 2 tubules utérins, l'intestin, le tissu musculaire, les lignes latérales et la cuticule dentelée $(\mathrm{H} / \mathrm{E}, \times 250)$.

4. Dentelure de la cuticule dans une femelle immature $(\mathrm{H} / \mathrm{E}, \times 500)$.

5. Section transversale de femelle gravide, isolée du nodule inflammatoire. Des fragments de microfilaires sont visibles dans les 2 tubules utérins $(\mathrm{H} / \mathrm{E}, \times 250)$.

6. Section transversale d'un mâle, mettant en évidence le testicule (tubule de gauche) et l'intestin $(\mathrm{H} / \mathrm{E}, \times 250)$.

7. Section transversale d'un mâle vers son extrémité caudale. On voit la présence des ailes caudales, du testicule et de l'intestin (PAS, $\times 250)$.

8. Section transversale d'un mâle à l'intérieur d'une artériole pulmonaire thrombosée (cas pulmonaire) (Masson-Goldner, $\times 100$ ). 
nulome formé étant détruit en quelques semaines sans attirer l'attention du patient. La mise au point d'une réaction sérologique spécifique et réellement sûre pourrait éclaircir cette hypothèse.

La parasitose chez l'homme a été signalée dans plus de la moitié des régions italiennes, apparemment avec une prévalence plus élevée dans les anciennes zones malariques du Nord (Vallée du Pô, régions de riziculture). Selon les expériences de Coluzzi (1964) et de Coluzzi et Trabucchi (1968), plusieurs espèces d'Anopheles et d'Aedes pourraient transmettre $D$. repens mieux que Culex spp., en relation avec l'absence ou la présence de l'armature bucco-pharyngée de l'insecte vecteur. Toutefois on n'a pas encore identifié avec certitude le vecteur naturel en Italie pour l'homme, ni pour le chien.

Il est difficile de donner une explication sur le nombre plus élevé de femmes parasitées que d'hommes : attention plus grande à une atteinte esthétique? terrain plus favorable à un développement des larves?

La fréquence plus élevée des cas au-dessus de 40 ans est également difficile à expliquer : affaiblissement de certaines réactions immunitaires en relation avec l'âge? Beaver et Orihel (1965) ont aussi noté, dans leurs cas observés aux USA et provoqués par d'autre filaires zoonotiques, le plus grand nombre de sujets atteints parmi les femmes et l'absence des cas dans l'enfance, mais sans pouvoir en donner une explication.

En conclusion, en dépit des progrès réalisés, plusieurs questions restent encore à éclaircir. La collaboration étroite des médecins avec les chirurgiens, les dermatologistes, les hispopathologistes, les immunologistes et les parasitologistes, est nécessaire pour les résoudre.

\section{RÉFÉRENCES}

Addario C. : Su di un nematode dell'occhio umano. Ann. Oftalmol., 1885, 14, 135-147.

Alessandrini G. : Nuovo caso di Filaria conjunctivae Add. parassita dell' uomo. Boll. R. Acc. Med. Roma, 1906, 32, 523-529.

Aloi F. G., Pippione M., Depaoli M. A. : Granuloma cutaneo da dirofilaria. G. It. Derm. Vener., 1983, 118, 377-385.

Babudieri B. : Un caso di filariosi nell' uomo, da Filaria conjunctivae Addario 1885, osservato in Italia. Riv. Parassit., 1937, 1, 53-67.

Balbo T., Panichi M. : La filariasi del cane. Epizoologia e diagnosi di laboratorio. N. Veter., 1968, 44, 78-92.

Bartoli C. : Focolaio infiammatorio solitario da filaria nel muscolo grande pettorale dell'uomo. Boll. Soc. It. Patologia., 1950, $1,129-130$.

Beaver P. C., Orihel Th. C. : Human infection with Filariae of animals in the United States. Am. J. Trop. Med. Hyg., 1965, 14, 1010-1029.

Bevacqua R., Wirth A. : Kyste de la cavité orbitaire dû à Filaria conjunctivae Addario. Ann. Parasit. Hum. Comp., 1950, 25, 37-41.

Bianchi L. : Filariasi nell' uomo : su quattro casi autoctoni osservati in provincia di Pavia. Rend. Sci. Ist. Lombardo, B, 1968, 102, 171-174.
Bianchi L., Caprioglio A. : Dirofilariosi sottocutanea nell' uomo. Sei casi autoctoni nella pianura padana. G. Mal. inf. parassit., 1972, 24, 98-104.

Bolgan A., Cassaro E., Turchetto M. : Due casi di dirofilaria negli annessi genitali maschili. G. Veneto Sci. Med., 1985, 36, 151-153.

Bonu G., Zina G. : Une rare affection chez l'homme : le granulome sous-cutané par Dirofilaria. Bull. Soc. Franç. Dermat. Syphil., 1972, 79, 248-250.

Cancrini G., Accardi A. : Un caso di dirofilariasi umana a Roma. Parassitologia, 1980, 22, 292-293.

Cancrini G., Scaglione F. : Osservazioni su due casi di dirofilariasi oculare umana in Sicilia. Parassitologia, 1984, 26, 273-282.

Cancrini G., Negro P., D'Amore L. : Un caso di dirofilariasi umana in Campania. Parassitologia, 1988, 30 (Suppl. 1), 36-37.

Cazzato G., Pivotti G., Candiani G. : Descrizione di una eccezionale localizzazione faringea di dirofilariasi. Otorinolaringoiatria, 1990, 40, 227-228.

Cirincione C. : Parassiti oculari. II. Filaria inermis Grassi, sottocongiuntivale. Clin. ocul., 1900, 2, 53-61.

Colla L., Fontana P. : Due casi di filariasi del sottocutaneo, autoctona, nell' uomo. Ateneo Parmense, 1967, 38, 192-198.

Coluzzi M. : Osservazioni sperimentali sul comportamento di Dirofilaria repens in diversi gruppi di artropodi ematofagi. Parassitologia, 1964, 6, 57-62.

Coluzzi M., Trabucchi R. : Importanza dell' armatura bucco faringea in Anopheles e Culex in relazione alle infezioni con Dirofilaria. Parassitologia, 1968, 10, 47-59.

Condorelli-Francaviglia M. : Filaria apapillocephala (mihi). Lo Spallanzani, 1892, 21, 109-120.

Dalla Pria A. F., Piaserico P. L., Piaserico G. P. : La dirofilariasi del sottocutaneo. Presentazione di un caso. Boll. Soc. med. chir. Modena, 1978, 78, 1-9.

De Carneri I., Sacchi S., Pazzaglia A. : Su sette casi accertati e su numerosi altri probabili di dirofilariosi del sottocutaneo nell' uomo, una zoonosi non rara in provincia di Pavia. Parassitologia, 1974, 16, 110-112.

Desportes C. : Filaria conjunctivae Addario 1885, parasite accidentel de l'homme, est un Dirofilaria. Ann. Parasit. hum. comp., 1939-1940, 17, 380-404, 515-532.

Dissanaike A. S. : Zoonotic aspects of filarial infections in man. Bull. WHO, 1979, 57, 349-357.

Dolfini G. : Un caso di filariosi autoctona nel Veneto. Minerva med., 1930, 21, 353-357.

Esposito R., Palmaro F., Galli C., Piffer R. : Un nuovo caso di dirofilariosi sottocutanea umana in Lombardia. G. Mal. inf. parassit., 1984, 36, 1059-1062.

Fabbretti G., Fedeli F., Alessi A., Boaron M., Salpietro V., Brisigotti M. : Human pulmonary dirofilariasis: report of a new European case. Histol. Histopath., 1990, 5, 311-313.

Faust E. C. : Human infection with species of Dirofilaria. Ztchr. Tropenmed. u. Parasit., 1957, 8, 59-68.

Foresi C., Caroli G. : Sulla dirofilariosi cutanea autoctona in Toscana. Arch. it. Sci. med. trop. e Parassit., 1973, 54, 41-50.

Fruttaldo L., Sarasso G., Saliva G., Guazzotti G., Carcò F. P. : Aspetti clinici ed epidemiologici di 4 casi di Dirofilariosi autoctona nel Vercellese. G. Mal. inf. parassit., 1985, 37, 1273-1275.

Gafà S., Morini C., Giudici M., D’Andrea L., Rossi G. : Un caso di dirofilariosi autoctona in provincia di Reggio Emilia. G. Mal. inf. parassit., 1983, 35, 761-763.

Garavelli P. L. : Dirofilariasi umana periorbitaria. Descrizione di un caso clinico. Minerva oftalmol., 1988, 30, 75-76.

Garavelli P. L., Sardi A., Raschio E. : Dirofilariasi autoctona in Alessandria. Minerva Med., 1990, 81 (suppl. 2), 59-60. 
Gardiner C. H., Oberdorfer C. E., Reyes J. E., Pinkus W. H. : Infection of man by Dirofilaria repens. Am. J. trop. Med. Hyg., 1978, 27, 1279-1281.

Gozzi B., Munafo' G., Saleri R., De Carneri I. : Un caso di dirofilariosi nell' uomo in provincia di Brescia. Parassitologia, 1980, $22,315-316$.

Grassi B. : Filaria inermis (mihi) ein Parasit des Menschen, des Pferdes und des Esels. Centrbl. Bakt. Parasitenk., I, 1887, 1, 617-623.

Guerra R., Casu L., Cavallini G. M. : Un caso di infezione orbitaria da Dirofilaria. Boll. Ocul., 1983, 62, 695-698.

Harant H., Brès A. : A propos de deux cas de parasitisme par filaires immatures. Bull. Soc. Path. exot., 1946, 39, 286-287.

Lampertico P., Bordoni U. : Filariosi cutanea. Istocitopatologia, 1979, 1, 9-12.

Locatelli A. : Sulle filariasi animali in provincia di Pavia. Parassitologia, 1971, 13, 197-202.

Malfatti G., Spaziante G., Torcigliani A. : La dirofilariosi del sottocutaneo. Ann. Sclavo, 1970, 12, 291-303.

Mantovani A. : Ricerche sulla infestazione da Dirofilaria repens Railliet e Henry 1911. N. Veter., 1967, 43, 180-189.

Marconcini A., Macchioni G., Cardini G., Braca C. : Indagine epidemiologica sulla filariasi del cane in Toscana. Atti Soc. it. Sci. vet., 1976, 30, 607-609.

Marconcini A., Molinari G., Paci A., Migliaccio P., Singer M. T., Marchetti G., Valentini P. : Osservazioni su un caso di dirofilariosi sottocutanea umana in provincia di Livorno. Microb. med., 1989, 4, 128-130.

Miracco C., Santopietro R., Vindigni C. : Dirofilariasi scrotale. Riv. Parassit., 1983, 44, 497-499.

Miracco C., Lorenzini L., Lio R., Luzi P. : Human dirofilariasis in Italy. Report of two new autoctonous cases in Tuscany. Riv. Parassit., 1987, 48, 265-269.

Pace A. : Sopra un nuovo nematode. G. Sci. nat. econ. R. Ist. tecn. Palermo, 1866, 2, 152-154.

Pampiglione S., Candiani G., Del Maschio O., Pavan V. : Dirofilariasi polmonare nell' uomo : un terzo caso in Italia. Pathologica, $1991 b, 83,21-27$.

Pampiglione S., Canestri-Trotti G., Piro S., Maxia C. : Dirofilariasi palpebrale nell' uomo : 1 caso in Sardegna. Pathologica, 1989, 81, 295-296.

Pampiglione S., Canestri-Trotti G., Rivasi F. : Quattordici nuovi casi di dirofilariasi umana in Italia. Pathologica, 1988, 80, 293-300.

Pampiglione S., Canestri-Trotti G., Squadrini F. : Human subcutaneous dirofilariasis. 2. A report of 5 new cases of Dirofilaria repens in Central and Northern Italy and of a sixth case with uncertain parasitological diagnosis. Parassitologia, 1982 b, 24, 167-176.

Pampiglione S., Fedeli F. : Dirofilariasi polmonare umana : aspetti parassitologici del $2^{\circ}$ caso segnalato in Italia. Parassitologia, 1991, 33 (sous presse).

Pampiglione S., Franco F., Canestri-Trotti G. : Human subcutaneous dirofilariasis. 1. Two new cases in Venice. Identification of the causal agent as Dirofilaria repens Raillet et Henry, 1911. Parassitologia, $1982 a$, 24, 155-165.

Pampiglione S., Manilla G., Canestri-Trotti G. : Dirofilariasi umana in Italia: un nuovo caso palpebrale con guarigione spontanea, in Abruzzo. Parassitologia, 1990, 32, 381-384.

Pampiglione S., Muretto P., Del Fiasco S. : Dirofilariasi sottocutanea umana in Italia : primo caso nelle Marche. Pathologica, $1991 a, 83,17-20$.

Pampiglione S., Poglayen G., Capelli G. : Distribuzione geografica della filariosi canina in Italia. Parassitologia, 1986, 28, 297-300.

Pampiglione S., Rivasi F., Canestri-Trotti G. : Human pulmonary dirofilariasis in Italy. Lancet, $1984 a$, Feb. 11: 333.

Pampiglione S., Rivasi F., Canestri-Trotti G. : Dirofilariosi polmonare umana : un caso in Italia. Pathologica, 1984b, 76, 565-572.

Pampiglione S., Rivasi F., Franco F. : Dirofilariasi sottocutanea umana : due nuovi casi nell' Italia del Nord. Parassitologia, $1991 c, 33$ (sous presse).

Pane C. : Nota su un elminto nematode. Ann. Accad. Aspiranti Natural. Napoli., 1864, 4, 32-34.

Pazzaglia A., Sacchi S., De Carneri I. : Un' affezione cutanea non rara in Italia : la dirofilariosi. Due nuovi casi pavesi. $G$. it. Dermat., 1974, 109, 218-224.

Pierantoni U. : Sulla Filaria labialis Pane 1864. Annuar. Mus. Zool. R. Univ. Napoli., 1908, 2, 1-5.

Pollavini G., De Lalla F. : Un caso autoctono di dirofilariosi sottocutanea nell' uomo. Ann. Sclavo, 1975, 17, 678-683.

Ricci M. : Un nuovo reperto di Dirofilaria conjunctivae (Addario, 1885) Desportes, 1939-40, nell' uomo in Italia. Riv. Parassit., 1963, 24, 73-79.

Rossi G. : Su di un caso di filariosi autoctona (Filaria conjunctivae Addario) a localizzazione premammaria. Arch. it. Chir., 1943, 64, 456-464.

Rossi G., Genesi M. : Pseudotumori parassitari. A proposito di quattro casi di nematodiasi autoctona da parassita filarioide. Minerva Med., 1953, 44, 1517-.1525

Scafa D., Viti A., Caianiello G. : La dirofilariasi sottocutanea dell' uomo. Descrizione di un caso nel Grossetano. G. Mal. Inf. Parassit., 1985, 37, 826-828.

Scaglia M., Senaldi G., Brustia R., Strosselli M., Gatti S. : Human subcutaneous dirofilariasis : a report of 4 new autochthonous cases in Italy and a review of the literature. Bull. Soc. Path. exot., 1985, 78, 629-636.

Skrjabin K. I., Althausen A. J., Schulman E. S. : First case of Dirofilaria repens in man. Trop. Med. Veter. Mosca., 1930, 8, 9-11 (cité par Faust, 1957).

Storace R. : Su di un rarissimo caso di Filariosi umana (da Filaria conjunctivae Addario, 1885) osservato in Italia. Arch. De Vecchi, 1963, 40, 711-730.

Supino F. : Sopra una Filaria dell' occhio umano. Atti Acc. Lincei. Rendic. Cl. fis. mat. nat., 1900, 9, 85-91.

Tecce P. : Sopra un caso di Filaria labialis (Pane). Riforma Med., 1908, 24, 933-934. 